\title{
Epilepsy in child, particularities related to evolution and prognosis - a case report and a review of the literature
}

\author{
Lorena Elena Melit', Cristina Oana Marginean' ${ }^{1}$, Andreea Dinca ${ }^{1}$, Raluca Damian², \\ Nicoleta Suciu ${ }^{3}$, Maria Oana Marginean ${ }^{3}$ \\ 1Pediatrics Clinic 1, Targu Mures, Romania \\ ${ }^{2}$ Neonatology Clinic 1, Targu Mures, Romania \\ ${ }^{3}$ University of Medicine and Pharmacy, Targu Mures, Romania
}

\begin{abstract}
Epilepsy is a brain pathology that consists in the development of recurrent epileptic seizures, and more than half of the cases present the onset during childhood. We present the case of a 3-year-old male patient, with heredocollateral antecedents of epilepsy and personal history of neuropsychomotor retardation, who was admitted in our clinic presenting generalized tonic clonic seizures, initially febrile and afterwards afebrile. The laboratory tests and the neuroimagistic ones did not identify an underlying cause. The initial EEG did not point out anything pathological. Despite the anticonvulsant treatment, the patient's evolution was unfavorable, presenting multiple short term recurrences, being readmitted multiple times in our clinic. Once we introduced the second anticonvulsant drug, levetiracetam, in his treatment, the seizures disappeared, and the patient presented long term favorable evolution. The case particularity consists in the development of generalized tonic clonic seizures in a patient with significant family history, and personal history of neuropsychomotor retardation that appear initial under febrile context, and afterwards in the lack of fever, repeating cyclic, with unfavorable evolution despite the treatment with valproic acid that disappear only after initiating treatment with levetiracetam.
\end{abstract}

Keywords: epilepsy, child, evolution, prognosis

\begin{abstract}
Abbreviations:
$\mathbf{C R P}$ - C-reactive protein $\quad \mathbf{E S R}$ - erythrocyte sedimentation rate

CT - computer tomography $\quad$ Leu - leukocytes

EEG - electroencephalogram W-weight
\end{abstract}

\section{INTRODUCTION}

The seizure is a paroxystic manifestation that comprises multiple signs and/or symptoms that result from the abnormal or synchronous activity of the neurons within the brain. According to the Internation Classification of Epileptic Seizures, these are divided into focal, previously called partial, and generalized (1). In case of focal epileptic seizures, the modifications of the electroencephalogram (EEG) suggest the aberrant neuronal activity to present the origin in one of the two cerebral hemispheres, while the generalized ones present an abnormal EEG which shows the involvement of both hemispheres in the abnormal activity. Febrile seizures represent acute manifestations, frequent in children, as a result of the brain excitability perturbation. The incidence of seizures, both febrile and afebrile, is up to $10 \%$ in the first years of life (1). On the other hand, $30 \%$ of the patients that present afebrile seizures will develop further on life epilepsy, the risk decreasing to $20 \%$ in case of a normal neurological exam, EEG and neuroimagistic after this first manifestation (1). Therefore, the development of a single febrile or afebrile seizure does not necessary establish the diagnosis of epilepsy. 
Epilepsy represents a cerebral pathology characterized by a persistent predisposition to develop seizures and their subsequent neurobiological, psychological and social consequences (1). The cumulated incidence of this pathology during life is of approximately $3 \%$, and more than a half of the cases present the onset during childhood, with a prevalence of $0.5-1 \%$ per year (1). The diagnosis of epilepsy involves the development of an unprovoked epileptic seizure associated either with another identical manifestation, either with enough clinical or EEG modifications to prove the persistent predisposition to develop recurrences (1). EEG is not mandatory in case of the first convulsive manifestation without other associated symptoms as it can also be normal in case of recurrences.

The first-line standard management in case of a patient with epilepsy is represented by the antiepileptic medication, and the drug itself will be chosen based on the type of epilepsy (2). Even though there are multiple guidelines that recommend a proper antiepileptic drug, in most of the cases, the management is still based on the approach of the therapeutic method, or 'trial-error' approach (3). Thus, the treatment of primary generalized tonic clonic seizures extended in the last 2 years, comprising new drugs, such as perampanel together with valproic acid, lamotrigine, levetiracetam, topiramat, while zonisamide and lacosamide are still studied (4).

\section{CASE REPORT}

We present the case of a 3-year-old male child, who was admitted in our clinic for generalized tonic clonic seizures, with a length of approximately 10-15 seconds and fixed view, associated with fever. The family history revealed the fact that the patients has a maternal cousin with epilepsy. The personal history underlined that the patient was diagnosed with a mild neuro-psychomotor retardation at the age of one year expressing a mild generalized hypotonia, for which he benefited from neurological treatment consisting in vitamins and gymnastics, and which, according to the neurologist, recuperated. The onset of the present disease was on the day of admission with a generalized tonic clonic seizure, presenting a length of approximately 10-15 seconds, with fixed view, in febrile context, therefore being admitted in our clinic for investigations and adequate treatment.

The clinical exam at the moment of admission pointed out the following pathological elements: mildly influenced general status, hyperemic phar- ynx, serous rhinorrhea, spastic cough, W: $15 \mathrm{~kg}$. The neurological exam did not reveal any major pathological elements besides drowsiness. The complete cellular blood count showed mild leukocytosis (Leu $10530 / \mathrm{mm}^{3}$ ), and the acute phase biomarker were increased (ESR $25 \mathrm{~mm} / \mathrm{h}$, CRP $25 \mathrm{mg} /$ dL). The peripheral blood smear suggested also the presence of a bacterial infection pointing out the predominance of polymorphonuclear cells (55\%). We also performed a brain CT and an EEG under emergency condition, which did not reveal anything pathological. On the day of admission, the patient repeated the seizure, therefore the neurologist recommended the initiation of the valproic acid syrup (Depakine) in a dose of $22 \mathrm{mg} / \mathrm{kg} /$ day and another EEG after 10 from the seizure. Thus, we established the diagnoses of febrile seizures and acute pharyngitis, and we administered antibiotics by vein for 5 days, antipyretics, but also the treatment recommended by the neurologist, with favorable evolution, discharging the patient after 5 days of hospitalization in good general status, without presenting seizures during admission.

After approximately 3 days from discharge, the seizures reappear, under both febrile conditions and in the lack of fever, therefore the mother presented with him to the neurologist who recommends the increase of Depakine dose to $31 \mathrm{mg} / \mathrm{kg} /$ day, but within the following 24 hours, the patient presents another 6 convulsive manifestations, therefore he is re-admitted in our clinic. The clinical exam and the laboratory test were within normal limits. We also performed a brain MRI, which showed adenoid hypertrophy, without other intracerebral injuries. During admission, we administered depletive treatment (Furosemide) and dexamethasone associated with Depakine, with favorable evolution, discharging the patient after approximately 3 days, with the recommendation to continue the treatment with Depakine and neurological monitoring.

The seizures reappear after 7 days from discharge, without presenting fever, associated with myoclonic manifestations during sleep, approximately 7 episodes within 24 hours, therefore the patient is admitted in the intensive care unit for stabilizing his status, being transferred in our clinic after approximately 24 hours. The laboratory test and the clinical exam did not reveal anything pathological, but the EEG pointed out typical sleep waves, "vertex spikes" and groups of hyper voltage theta waves, bilateral wave-spikes complexes on the frontal and central derivations, therefore we established the diagnosis of generalized tonic clonic epilepsy and we associated levetiracetam in the 
treatment, with favorable evolution. After approximately 6 months, the patient was seizures-free, therefore the valproic acid was progressively reduced, remaining only on levetiracetam treatment, with favorable evolution.

The case particularity consists in the development of generalized tonic clonic seizures in a patient with significant family history, and personal history of neuropsychomotor retardation that appear initial under febrile context, and afterwards in the lack of fever, repeating cyclically, at approximately 7-10 days, with unfavorable evolution despite the treatment with valproic acid that disappear only after initiating treatment with levetiracetam.

\section{DISCUSSIONS}

Epilepsy is a neurological pathology that affects approximately 50 million people worldwide (5), with long term devastating implications regarding both the individual and the society. The highest incidence of epilepsy is within the first 2 years of life, afflicting between 56 and 88/100,000 children/year leading to the increase of neurological and behavioral impairment risk in these patients (3). Similarly, in case of our patient, the onset was also at a small age. It is well-documented the fact that the early diagnosis and control of epileptic seizures are associated with better long term outcome regarding the neurological development of these children, but most of epilepsies with onset at a small age present a bad prognosis regarding the control of convulsive manifestations (6-8). Thus, it seems that approximately $20-40 \%$ of the patients diagnosed with epilepsy continue to present seizures despite the administered pharmacological therapy, category of patients that suffer from so called epilepsy refractory to treatment (9). In the case of the above mentioned patient, even though the initial evolution was burdened by multiple convulsive recurrences, once we introduced levetiracetam in the treatment, he no longer presented seizures. Approximately $80 \%$ of the cases diagnosed with epilepsy are encountered in developing countries, where this pathology remains a major public health problem
(10). The life quality for patients diagnosed with epilepsy is burdened by poor social insertion due to fear, misunderstanding, wrong believes, the lack of knowledge and social discrimination (11). Most of epilepsies diagnosed at small ages cannot be classified into an epileptic syndrome, remaining unclear, weakly defined, entities, whose cause cannot be identified (2).

The pharmacological therapy is the first choice in the management of patients diagnosed with epilepsy. Thus, there are multiple antiepileptic drugs available currently, such as: valproic acid, lamotrigine, levetiracetam, topiramat etc. Recent studies discovered also another two compounds, zonisamide and lacosamide useful for controlling epileptic seizures, but which need further studies in order to be available for the current medical use (4). Recent data suggest that in case of generalized tonic clonic seizures in children with possible cognitive problems, valproic acid would not have promising results, while lamotrigine or levetiracetam represent two viable options for this group of patients (4). In case of our patient, who present history of neuropsychomotor retardation, the convulsive manifestations could not be controlled only after the introduction of levetiracetam in the treatment. Due to the increase rate of seizures recurrence despite the adequate pharmacological therapy, nutritional therapies represent an option worth to be explored in case of epilepsies refractory to treatment. Despite all these available therapeutic methods, patients diagnosed with epilepsy at small ages present the highest risk for recurrences or poor response to treatment.

\section{CONCLUSIONS}

Epilepsy represents a chronic neurological pathology, with unpredictable evolution and poor prognosis, especially in case of early onset. Levetiracetam represents a viable option for children with epilepsy that do not respond to the initial treatment. Despite all these facts, small age at onset represents a major factor of poor long-term prognosis for patients diagnosed with epilepsy.

\section{REFERENCES}

1. Mikati M.A., Hani A.J. Seizures in Childhood. In Kliegman RM, Stanton BF, St. Geme III JW, Schor NF. Nelson Texbook of Pediatrics, Edition 20. Philadelphia: Elsevier, 2016:2823-2831.

2. Titre-Johnson S., Schoeler N., Eltze C. et al. Ketogenic diet in the treatment of epilepsy in children under the age of 2 years: study protocol for a randomised controlled trial. Trials 2017; 18:195.

3. Eltze C.M., Chong W.K., Cox T. et al. A population-based study of newly diagnosed epilepsy in infants. Epilepsia 2013; 54:437-45.

4. Coppola G., Piccorossi A., Operto F.F., Verrotti A. Anticonvulsant drugs for generalized tonic-clonic epilepsy. Expert Opin Pharmacother 2017. doi: 10.1080/14656566.2017.1328499 [Epub ahead of print].

5. World Health Organization - WHO. Epilepsy. Geneva: World Healh Organization 2016. Disponível em:http://www.who.int/mediacentre/ factsheets/fs999/en/ 
6. Freitag H., Tuxhorn I. Cognitive function in preschool children after epilepsy surgery: rationale for early intervention. Epilepsia. 2005; 46:561-567.

7. Chevrie J.J., Aicardi J. Convulsive disorders in the first year of life: neurological and mental outcome and mortality. Epilepsia. 1978; 19:67-74.

8. Deonna TaR-P.E. Cognitive and behavioural disorders of epileptic origin in children. Mac Keith Press 2005.
9. Kwan P., Brodie M.J. Early identification of refractory epilepsy. N Engl J Med 2000; 342(5):314-319. https://doi.org/10.1056/ NEJM200002033420503.

10. Henok A., Lamaro T. Knowledge about and Attitude towards Epilepsy among Menit Community, Southwest Ethiopia. Ethiop J Health Sci 2017; 27(1):47-58.

11. Aldenkamp A. et al. Newer antiepileptic drugs and cognitive issues. Epilepsia. 2003; 44(suppl 4):21-29. 